\title{
In vivo evaluation of patellar tendon stiffness in individuals with patellofemoral pain syndrome
}

\author{
Hsin-Yi Liu ${ }^{\mathrm{a}, \mathrm{b}, \mathrm{c} *}$, Michelle Boling ${ }^{\mathrm{b}}$, Darin Padua ${ }^{\mathrm{b}}$, R. Alexander Creighton ${ }^{\mathrm{a}}$ and Paul Weinhold ${ }^{\mathrm{a}, \mathrm{b}}$ \\ ${ }^{a}$ Department of Orthopaedics, University of North Carolina, Chapel Hill, NC 27599, USA; ${ }^{b}$ Sports Medicine Laboratory, University of \\ North Carolina, Chapel Hill, NC 27599, USA; ' Department of Physical Education and Recreation, North Carolina Central University, \\ Durham, NC 27707, USA
}

(Received 21 April 2008; final version received 29 October 2008)

\begin{abstract}
The objective of this study was to utilise an ultrasonic technique to assess the effect of patellofemoral pain syndrome (PFPS) on the mechanical properties of the patellar tendon. Seven subjects with PFPS and seven matched control subjects volunteered to participate in this study. Subjects were asked to perform isometric maximal voluntary contractions of the knee extensors while their knee extension torque was monitored and the displacement of the patellar tendon was recorded with an ultrasonic system. Our results showed significantly lower tendon stiffness (by 30\%) in the PFPS subjects. Although tendon secant modulus was lower by $34 \%$ in the PFPS subjects, the difference was not statistically significant. Therefore, we conclude that the ultrasonic technique was able to detect a decrease in the structural stiffness of the patellar tendon associated with PFPS. The decrease in tendon stiffness was moderately correlated with the length of symptoms in these individuals.
\end{abstract}

Keywords: tendon mechanical properties; ultrasound measurement; anterior knee pain; tendon elastic modulus; chondromalacia patella, knee joint loading

\section{Introduction}

The evaluation of the mechanical properties of tendons has been commonly used as an objective means to describe the functional and recovery level of tendons in animal models (Bruns et al. 2000; Butler et al. 2004; Jackson et al. 1993). However, due to the invasive nature of the traditional method to evaluate the mechanical properties of tendons, it has been very difficult to apply in humans. A newly developed real time ultrasound imaging technique has been applied to study the mechanical properties of human tendons in vivo. To date, this technique has been successfully used to evaluate the effect of complete disuse and strengthening exercise on tendon stiffness (Kubo et al. 2004; Maganaris et al. 2006). These findings in humans were similar to those reported from animal investigations.

Patellofemoral pain syndrome (PFPS) is a common diagnosis of knee pain (Devereaux and Lachmann 1984; Fredericson and Yoon 2006; Piva et al. 2006; Thomee et al. 1999), which is usually associated with significant pain, and a limited activity level of the symptomatic limb. However, the success of the rehabilitative treatments for PFPS remains controversial because of the subjective content of commonly used clinical evaluation methods, such as pain scales and functional activity questionnaires (Arroll et al. 1997; Avraham et al. 2007; Bizzini et al. 2003; Boling et al. 2006; Lun et al. 2005; Thomee et al. 1996; Witvrouw et al. 2000). Strength assessments have been one method of objectively evaluating outcomes after rehabilitation treat- ments (Herrington and Al Sherhi 2007; Witvrouw et al. 2000). Previous studies of PFPS have reported decreased knee extensor or lower limb torques (Callaghan and Oldham 2004; Werner 1995) for the symptomatic limb compared to the asymptomatic limb or compared to the limbs of control subjects. Altered activity of the affected limb due to pain may contribute to these strength deficits in PFPS patients. Previous animal studies suggest that immobilization of a limb alters the mechanical properties of tendons (Atkinson et al. 1998; Kasperczyk et al. 1991). The effects of physical activity limitations in individuals with PFPS on the mechanical properties of the patellar tendon have yet to be investigated. An in vivo ultrasonic technique to assess the change in tendon properties may be a useful addition to conventional methods for assessing the efficacy of rehabilitation in individuals with PFPS. In order to determine if the capability of this ultrasonic technique may help in determining the efficacy of interventions, the first step is to assess whether a difference of tendon properties exists between individuals with PFPS and healthy individuals. Therefore, the purpose of this study was to utilize the ultrasonic technique to assess the effect of PFPS on the mechanical properties of the patellar tendon.

\section{Methods}

Seven subjects with PFPS (4 males and 3 females, age: $26 \pm 4$ years old, height: $176 \pm 10 \mathrm{~cm}$, body mass:

*Corresponding author. Email: hliu@nccu.edu

ISSN: 1176-2322 print/ 1754-2103 online 
Table 1. Demographic data, visual analogue scale (VAS) and the international knee documentation committee (IKDC) subjective knee evaluation scores of subjects.

\begin{tabular}{|c|c|c|c|}
\hline Parameter & PFPS & Control & $p$ \\
\hline Age (years) & $26 \pm 4$ & $27 \pm 4$ & $p=.36$ \\
\hline Height $(\mathrm{cm})$ & $176 \pm 10$ & $177 \pm 9$ & $p=.19$ \\
\hline Body mass $(\mathrm{kg})$ & $79 \pm 21$ & $79 \pm 22$ & $p=.34$ \\
\hline VAS* & $5.21 \pm 2.55$ & $0.11 \pm 0.20$ & $p=.002$ \\
\hline IKDC* & $56.16 \pm 6.41$ & $96.22 \pm 5.34$ & $p<.001$ \\
\hline
\end{tabular}

*VAS is scored by calculating the ratio of the length from the vertical mark to the point indicated no pain at all and the total length of the horizontal line, and then transforming the score to a scale that ranges from 0 to 10 . The IKDC subjective knee evaluation form is scored by summing the scores for the individual items and then transforming the score to a scale that ranges from 0 to 100 .

$79 \pm 21 \mathrm{~kg}$ ) and seven age, gender and weight-matched control subjects (4 males and 3 females, age: $27 \pm 4$ years old, height: $177 \pm 9 \mathrm{~cm}$, body mass: $79 \pm 22$ $\mathrm{kg}$ ) volunteered to participate (Table 1). Inclusion criteria for the patellofemoral pain group included: (1) anterior or retropatellar knee pain present during at least two of the following activities- ascending/descending stairs, hopping/running, squatting, kneeling and prolonged sitting; (2) insidious onset of symptoms not related to trauma; (3) pain on palpation of patellar facets and (4) worst pain in the past week greater than $3 \mathrm{~cm}$ on a $10 \mathrm{~cm}$ visual analogue scale. Inclusion criteria were adapted from Cowan et al. (2002).
Exclusion criteria for the patellofemoral pain and control groups included: (1) history of knee surgery; (2) clinical evidence of other knee pathology and (3) current significant injury affecting other lower extremity joints. All subjects signed an informed consent form that was approved by the University's institutional review board. Before the testing, each participant was instructed as how to fill out a $10-\mathrm{cm}$ visual analogue scale (VAS) (Chesworth and Vandervoort 1989), International Knee Documentation Subjective Knee Form (IKDC) (Irrgang et al. 2001) according to their experiences in pain level at the knee and for functional activities in the past two weeks prior to their testing date, and reported the length of their PFPS symptoms in months prior to testing.

Subjects were asked to perform five isometric maximal voluntary contractions (MVIC) of the knee extensors at $90^{\circ}$ of knee and hip flexion while seated and secured in an isokinetic dynamometer (Kin-Com 125E Plus, Harrison, TN). A load cell attached to the ankle cuff just above the medial malleolus was used to acquire the force produced during the tasks at $1000 \mathrm{~Hz}$. The product of the force data and the length of the lower leg measured from knee joint centre to the centre of the cuff was then used to calculate the knee resultant moment. During the MVIC task, a 7.5 MHz lineararray ultrasound probe (Compact System Diasonics, Santa Clara, CA) was secured to the knee to record the displacement of the inferior border of the patella at $30 \mathrm{~Hz}$ (Figure 1). A silicon template was taped onto the skin over the patella-

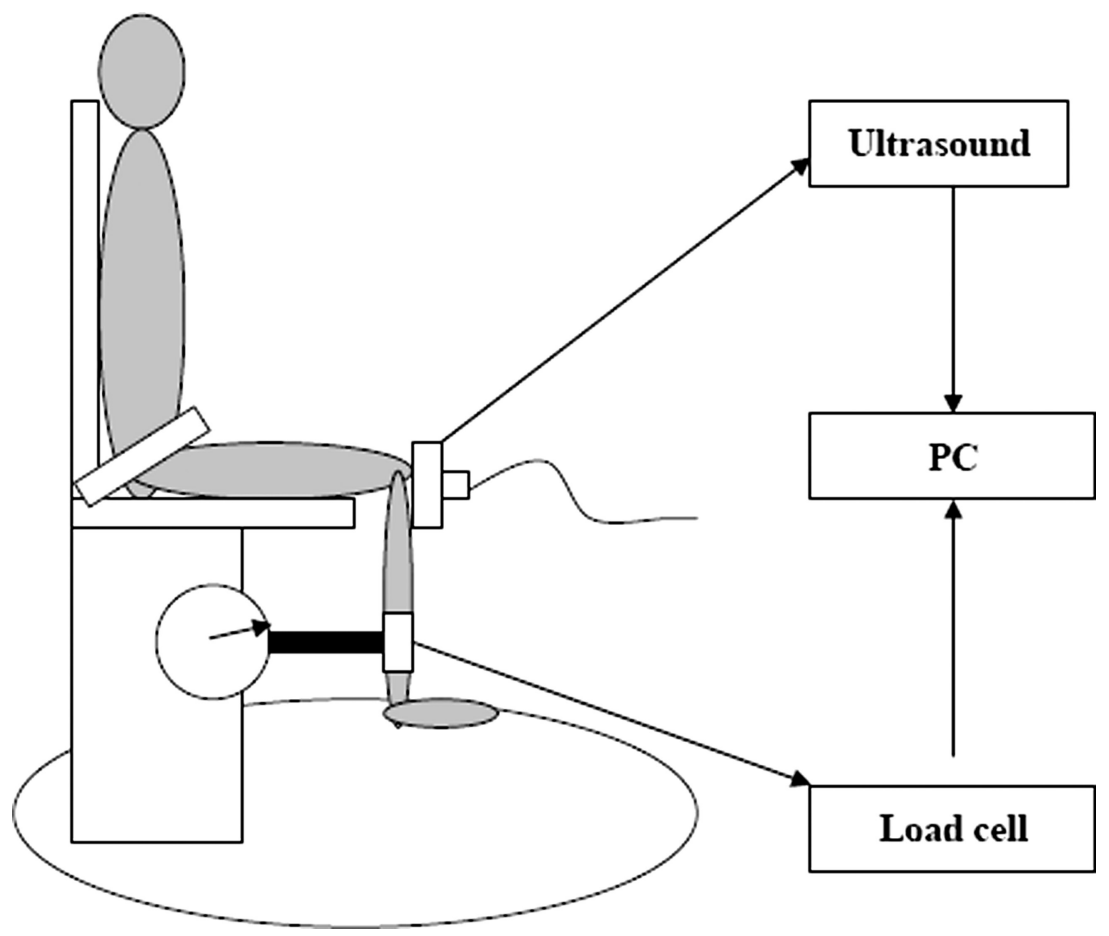

Figure 1. Experimental set-up: the ultrasound transducer was placed at the inferior pole of the patella, and the load cell was attached to the ankle cuff that was fixed to the Kin-com dynamometer system. The real-time ultrasound images and load cell signals were collected with an IBM laptop. 
tendon area to prevent sliding of the ultrasound probe. An echoabsorptive band was attached to the skin over the scan region to help monitor any sliding between the skin and the ultrasound probe. The force and ultrasound video data acquisitions were synchronized with an electronic trigger and recorded on an IBM computer. A non-telemetered surface electromyographic (EMG) system (Delsys Bagnoli-8, Boston, MA) was used to assess the muscle activity of the medial hamstring musculature at $1440 \mathrm{~Hz}$. Surface electrodes were positioned in parallel over the area of greatest bulk of the medial or lateral hamstrings. The EMG activity of the hamstrings were monitored during both the extension task and a subsequent isometric knee flexion task at $90^{\circ}$. The ratio of the hamstring EMG during extension to a maximal flexion task was used to calibrate the co-contraction effect during the MVIC extension tasks. The product of the ratio of the hamstrings EMG and the maximal knee flexion moment was used to adjust the knee resultant moment to estimate the net moment produced by the patellar tendon. The patellar tendon moment was then divided by the patellar tendon moment arm to calculate the patellar tendon force. The figure demonstration and equations of how we calibrated the co-contraction effect with hamstrings EMG data and calculated the patellar tendon force are described in Figure 2. The length of the moment arm of the patellar tendon was estimated from the thigh length and the knee flexion angle of each subject as described by Visser et al. (1990).

The ultrasound video of the patella-tendon movement was extracted into a series of 8-bit bitmap images $(640 \times$ 480 pixels) with an image-extracting program. These digital images were then analysed with a pattern matching technique based on a two-dimensional cross-correlation algorithm (IMAQ Vision builder 6; National Instruments, Austin, TX) to determine the coordinates of the patella frame by frame in the ultrasound image. The tendon deformation was calculated by subtracting the $\mathrm{X}$ coordinates of the patella at the first frame when the knee was relaxed from the $\mathrm{X}$ coordinates of the patella in each subsequent frame. The displacements of the patella in Y direction were ignored due to their minimal effects on the total displacements. The maximal tendon deformation was determined as the maximal displacement of the patella during the knee extension task. The linear force data and the patellar tendon deformation were used to plot a force-deformation curve. Each tendon's maximal force level and the corresponding deformation were chosen as the points of interest; then the slope of a line drawn from the point of interest to the origin of the force-deformation curve was used to calculate the structural stiffness.

Using a transverse ultrasonic image and a pair of longitudinal ultrasonic images, respectively, the cross-sectional area $(\mathrm{CSA})\left(\mathrm{mm}^{2}\right)$ and original length of the patellar tendon $(\mathrm{mm})$ were measured with the IMAQ Vision Builder software and converted to metric units. The structural stiffness

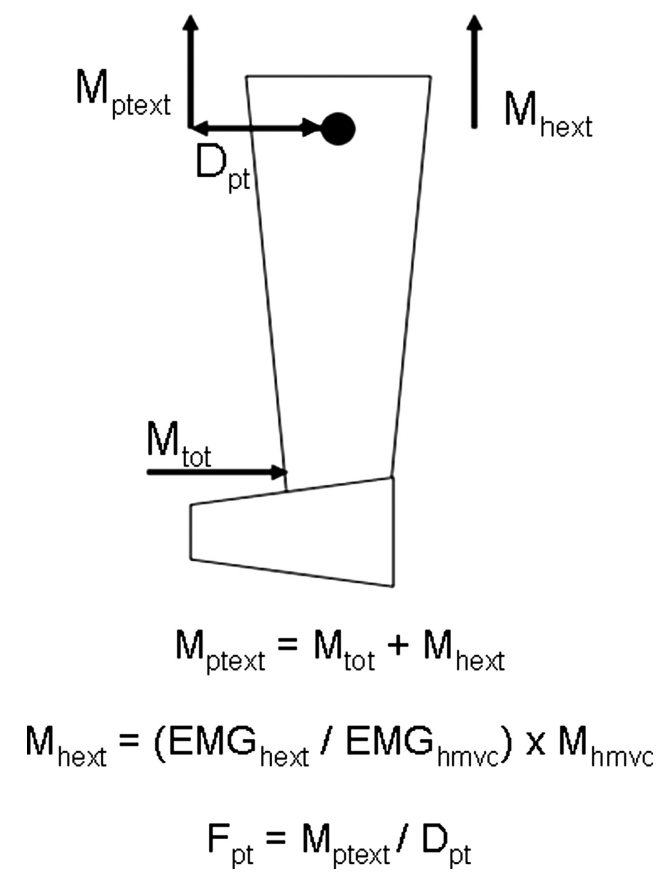

Figure 2. $\mathrm{M}_{\text {hext }}$ is the torque produced by hamstrings during the MVIC extension task; $\mathrm{M}_{\mathrm{hmvc}}$ is the torque produced by the hamstrings during MVIC flexion task; $\mathrm{EMG}_{\text {hext }}$ is the hamstrings EMG activity during the MVIC extension task; $\mathrm{EMG}_{\mathrm{hmvc}}$ is the RMS EMG activity during the MVIC flexion task; $M_{\text {ptext }}$ is the torque produced by the patellar tendon during the MVIC extension task; $\mathrm{M}_{\mathrm{tot}}$ is the resultant knee torque during the MVIC extension task; $F_{p t}$ is the force produced by the patellar tendon; $D_{p t}$ is the moment arm of the patellar tendon.

was multiplied by the ratio of the original length to the CSA to compute the material stiffness (secant modulus) of the tendon.

The demographic data, knee function questionnaire scores and biomechanical parameters (tendon length, tendon CSA, max. tendon force, max. tendon deformation, tendon strain, tendon stress, stiffness and secant modulus) of the PFPS knees were compared with the knee data of the matched control group, using a paired $t$ test with a significance level of $p<.05$. The correlation between length of symptoms and decrease in tendon stiffness was assessed with the Spearman correlation test $(p<.05)$. The assumptions of normality and equality of variance were met with all the measured variables, except for the measures of length of symptoms and decreased in tendon stiffness.

\section{Results}

There were no significant differences between the two groups in age, weight or height (Table 1). However, our PFPS participants had significant higher VAS scores and significantly lower functional activity scores compare to their matched control subjects (Table 1). The resting length 
Table 2. Mechanical and material properties evaluated.

\begin{tabular}{lccc}
\hline Parameter & PFPS & Control & $p$ \\
\hline Tendon length (mm) & $46 \pm 9$ & $52 \pm 15$ & $p=.190$ \\
Tendon CSA (mm $\left.{ }^{2}\right)$ & $85 \pm 13$ & $92 \pm 16$ & $p=.140$ \\
Max. tendon force (N) & $4906.48 \pm 1513.15$ & $6225.42 \pm 1577.25$ & $p=.005$ \\
Max. tendon & $2.70 \pm 0.81$ & $2.68 \pm 0.96$ & $p=.940$ \\
$\quad$ deformation (mm) & & & \\
Tendon strain (\%) & $6.55 \pm 2.14$ & $4.62 \pm 1.79$ & $p=.060$ \\
Tendon stress (MPa) & $54 \pm 21$ & $66 \pm 19$ & $p=.350$ \\
Stiffness (N/mm) & $1735 \pm 735$ & $2477 \pm 568$ & $p=.030$ \\
Secant modulus (GPa) & $1.04 \pm 0.59$ & $1.57 \pm 0.76$ & $p=.090$ \\
\hline
\end{tabular}

and CSA of the patellar tendon were similar in the groups (Table 2). Tendon stiffness was found to be significantly decreased by $30 \%$ in the PFPS subjects compared with the control subjects (Table 2). The maximal tendon force during isometric knee extension in the control group was significantly higher than the PFPS group (Table 2), however, the maximal tendon deformations were similar between the two groups (Table 2). The maximal stress and strain levels during isometric knee extension were not significantly different between the groups (Table 2). Although tendon modulus was lower by $34 \%$ in the PFPS subjects compared with the control subjects, the difference was not statistically significant (Table 2). The average length of symptoms for the PFPS subjects was $43 \pm 34$ months. A significant correlation was found between tendon stiffness and the length of symptoms $(r=0.75)$.

\section{Discussion}

Previous studies have reported the patellar tendon stiffness of healthy subjects ranging from 1790 to $1904 \mathrm{~N} / \mathrm{mm}$ (age: $24 \pm 3$ years) to 3725 to $4334 \mathrm{~N} / \mathrm{mm}$ (age: $30 \pm 7$ years) (Hansen et al. 2006; Yasuda and Hayashi 1999). The stiffness values of the healthy group observed in this study were within this range. The results of this study showed the structural stiffness of the patellar tendon to be decreased significantly in subjects with PFPS relative to matched-controls. The significant decrease in tendon stiffness in our PFPS group can result from a combination of material changes of the tendon or geometry changes (length and CSA). The smaller CSA and decreased secant modulus observed for the PFPS group would both act to decrease the tendon stiffness; however, neither of these measures on their own were significantly different between the groups. The trend in the PFPS group for a decrease in the secant modulus, a material property, would suggest that material changes may be making a larger contribution to the observed decrease in tendon stiffness in the PFPS group.

Our results coincide with similar changes in tendon properties reported in previous animal studies of disuse (Atkinson et al. 1998; Kasperczyk et al. 1991). On the basis of those studies, the reduction in mechanical properties of tendons could be due to the reduction of the total area of collagen fibrils in the tendon cross-section and the increase in the numbers of thin and immature fibrils. The functional activity level of our PFPS subjects was significantly decreased compared to the control group, which was also shown in previous studies (Hahn and Foldspang 1998); therefore, we speculate the decrease in tendon stiffness is a result of the decreased activity that often occurs with PFPS.

In addition, PFPS symptom length and the decrease in tendon stiffness were found to be moderately correlated. We speculate that due to individuals with PFPS reporting on average 3.5 years of PFPS symptoms, the limitations in physical activity for this period of time caused a decrease in tendon stiffness. Future studies with larger sample sizes and prospective data on length of symptoms are needed to better characterize this relationship.

Our study results differ from a recently reported study by Wilson et al. (2008). Their results suggested that PFPS subjects had significantly greater vastus medialis obliquus (VMO) tendon stiffness than their control subjects. However, during the sub-maximal voluntary quadriceps contractions performed in their study the resultant force present in each of the components of the quadriceps muscle group could not be determined, but were assumed to be uniform. With this assumption a decrease in the deformation of the VMO tendon might be interpreted as an increase in tendon stiffness, while it may also be a result of decreased force being transferred through the VMO component (Miller et al. 1997; Powers et al. 1996). Thus, an overestimation of the tendon force on the VMO tendon could have caused the significant increase in their stiffness measurement.

Our finding of decreased tendon stiffness in patients with PFPS has clinical relevance in two regards. With further study we conjecture that tendon stiffness measurements may prove to provide a more objective assessment of the extent of loading of the knee extensor apparatus in PFPS patients than muscle strength evaluations which may be more susceptible to momentary episodes of pain that may cause muscle inhibition and variability in strength testing results. While our hypothesis is that the observed decreased tendon stiffness in PFPS patients is a result of decreased physical activity of the symptomatic limb, it is also possible that decreased tendon stiffness could be a predisposing risk factor for the development of PFPS.

One limitation of this investigation was the restriction to two-dimensional images and the imperfect external fixation of the tibia. In future studies a device for monitoring the movement of the tibia will be applied to exclude any error in tendon displacement due to unexpected movements of distal end of the patellar tendon. Another limitation was the method used for calculating tendon stiffness. With the secant method of calculating tendon stiffness, it was possible that observed differences in tendon stiffness and modulus between groups could be caused by differences in maximal stress levels or the extent of the toe region; however, due 
to the similar stress and strain levels of the two groups, the likelihood of these effects influencing our measurement appeared minimal.

In order for this non-invasive ultrasonic technique to be used to evaluate the efficacy of rehabilitative therapies for PFPS, future studies need to assess whether this technique is able to predict the improvement in patients with PFPS. In addition, efforts towards simplifying the operation and analysis procedures of the ultrasonic technique should be made in the future so that this technique can be easily performed and applicable for clinical use.

\section{References}

Arroll B, EllisPegler E, Edwards A, Sutcliffe G. 1997. Patellofemoral pain syndrome - A critical review of the clinical trials on nonoperative therapy. Am J Sports Med. 25:207212.

Atkinson TS, Atkinson PJ, Mendenhall HV, Haut RC 1998. Patellar tendon and infrapatellar fat pad healing after harvest of an ACL graft. J Surg Res. 79:25-30.

Avraham F, Aviv S, Ya'akobi P, Faran H, Fisher Z, Goldman Y, Neeman G, Carmeli E. 2007. The efficacy of treatment of different intervention programs for patellofemoral pain syndrome - A single blinded randomized clinical trial. Pilot study. TSWJ 7:1256-1262.

Bizzini M, Childs JD, Piva SR. 2003. Systematic review of the quality of randomized controlled trials for patellofemoral pain syndrome. J Orthop Sports Phys Ther. 33:4-20.

Boling MC, Bolgla LA, Mattacola CG, Uhl TL, Hosey RG. 2006. Outcomes of a weight-bearing rehabilitation program for patients diagnosed with patellofemoral pain syndrome. Arch Phys Med Rehabil. 87:1428-1435.

Bruns J, Kampen J, Kahrs J, Plitz W. 2000. Achilles tendon rupture: experimental results on spontaneous repair in a sheep-model. Knee Surg Sports Traumatol Arthrosc. 8:364-369.

Butler DL, Juncosa N, Dressler MR. 2004. Functional efficacy of tendon kepair processes. Annu Rev Biomed Eng. 6:303329.

Callaghan MJ, Oldham JA. 2004. Quadriceps atrophy: to what extent does it exist in patellofemoral pain syndrome? Br J Sports Med. 38:295-299.

Chesworth BM, Vandervoort AA. 1989. Age and passive ankle stiffness in healthy women. Phys Ther. 69:217-224.

Cowan SM, Hodges PW, Bennell KL, Crossley KM. 2002. Altered vastii recruitment when people with patellofemoral pain syndrome complete a postural task. Arch Phys Med\& Rehabil. 83:989-995.

Devereaux MD, Lachmann SM. 1984. Patello-femoral arthralgia in athletes attending a sports injury clinic. Br J Sports Med. $18: 18-21$.

Fredericson M, Yoon KS. 2006. Physical examination and patellofemoral pain syndrome. Am J Phys Med \& Rehabil. 85:234-243.

Hahn T, Foldspang A. 1998. Prevalent knee pain and sport. Scand J Publ Health. 26:44-52.

Hansen P, Bojsen-Moller J, Aagaard P, Kjaer M, Magnusson SP. 2006. Mechanical properties of the human patellar tendon, in vivo. Clin Biomech. (Bristol, Avon) 21:54-58.
Herrington L, Al Sherhi A. 2007. A controlled trial of weight-bearing versus non-weight-bearing exercises for patellofemoral pain. J Ortho Sports Phys Ther. 37:155-160.

Irrgang JJ, Anderson AF, Boland AL, Harner CD, Kurosaka M, Neyret P, Richmond JC, Shelborne KD. 2001. Development and validation of the international knee documentation committee subjective knee form. Am J Sports Med. 29:600-613.

Jackson DW, Grood ES, Goldstein JD, Rosen MA, Kurzweil PR, Cummings JF, Simon TM. 1993. A comparison of patellar tendon autograft and allograft used for anterior cruciate ligament reconstruction in the goat model. Am J Sports Med. 21:176-185.

Kasperczyk WJ, Bosch U, Oestern HJ, Tschcerne H. 1991. Influence of immobilization on autograft healing in the knee joint. A preliminary study in a sheep knee PCL model. Arch Orthop Trauma Surg. 110:158-161.

Kubo K, Akima H, Ushiyama J, Tabata I, Fukuoka H, Kanehisa H, Fukunaga T. 2004. Effects of 20 days of bed rest on the viscoelastic properties of tendon structures in lower limb muscles. Br J Sports Med. 38:324-330.

Lun VMY, Wiley JP, Meeuwisse WH, Yanagawa TL. 2005. Effectiveness of patellar bracing for treatment of patellofemoral pain syndrome. Clin J Sport Med. 15:233-238.

Maganaris CN, Reeves ND, Rittweger J, Sargeant AJ, Jones DA, Gerrits K, De Haan A. 2006. Adaptive response of human tendon to paralysis. Muscle Nerve. 33:85-92.

Miller JP, Sedory D, Croce RV. 1997. Leg rotation and vastus medialis oblique vastus lateralis electromyogram activity ratio during closed chain kinetic exercises prescribed for patellofemoral pain. J Athl Train. 32:216-220.

Piva SR, Fitzgerald K, Irrgang JJ, Jones S, Hando BR, Browder DA, Childs D. 2006. Reliability of measures of impairments associated with patellofemoral pain syndrome. BMC Musculoskeletal Disord. 7.

Powers CM, Landel R, Perry J. 1996. Timing and intensity of vastus muscle activity during functional activities in subjects with and without patellofemoral pain. Phys Ther. 76:946-955.

Thomee R, Augustsson J, Karlsson J. 1999. Patellofemoral pain syndrome - A review of current issues. Sports Med. 28:245262.

Thomee R, Grimby G, Svantesson U, Osterberg U. 1996. Quadriceps muscle performance in sitting and standing in young women with patellofemoral pain syndrome and young healthy women. Scand.J Med Sci. Sports 6:233-241.

Visser JJ, Hoogkamer JE, Bobbert MF, Huijing PA. 1990. Length and moment arm of human leg muscles as a function of knee and hip-joint angles. Euro J Appl Phys. 61:453-460.

Werner S. 1995. An evaluation of knee extensor and knee flexor torques and EMGs in patients with patellofemoral pain syndrome in comparison with matched controls. Knee Surg Sports Traumatol Arthrosc. 3:89-94.

Wilson NA, Press JR, Zhang L. 2008. In vivo comparison of tendon mechanical properties in patellofemoral pain. Poster session presented at: 2008 ORS. 54th Annual Meeting of the Orthopaedic Research Society; San Francisco, California.

Witvrouw E, Lysens R, Bellemans J, Peers K, Vanderstraeten G. 2000. Open versus closed kinetic chain exercises for patellofemoral pain: a prospective, randomized study. Am J Sports Med. 28:687-694.

Yasuda K., Hayashi K. 1999. Changes in biomechanical properties of tendons and ligaments from joint disuse. Osteoarthritis Cartilage. 7:122-129. 

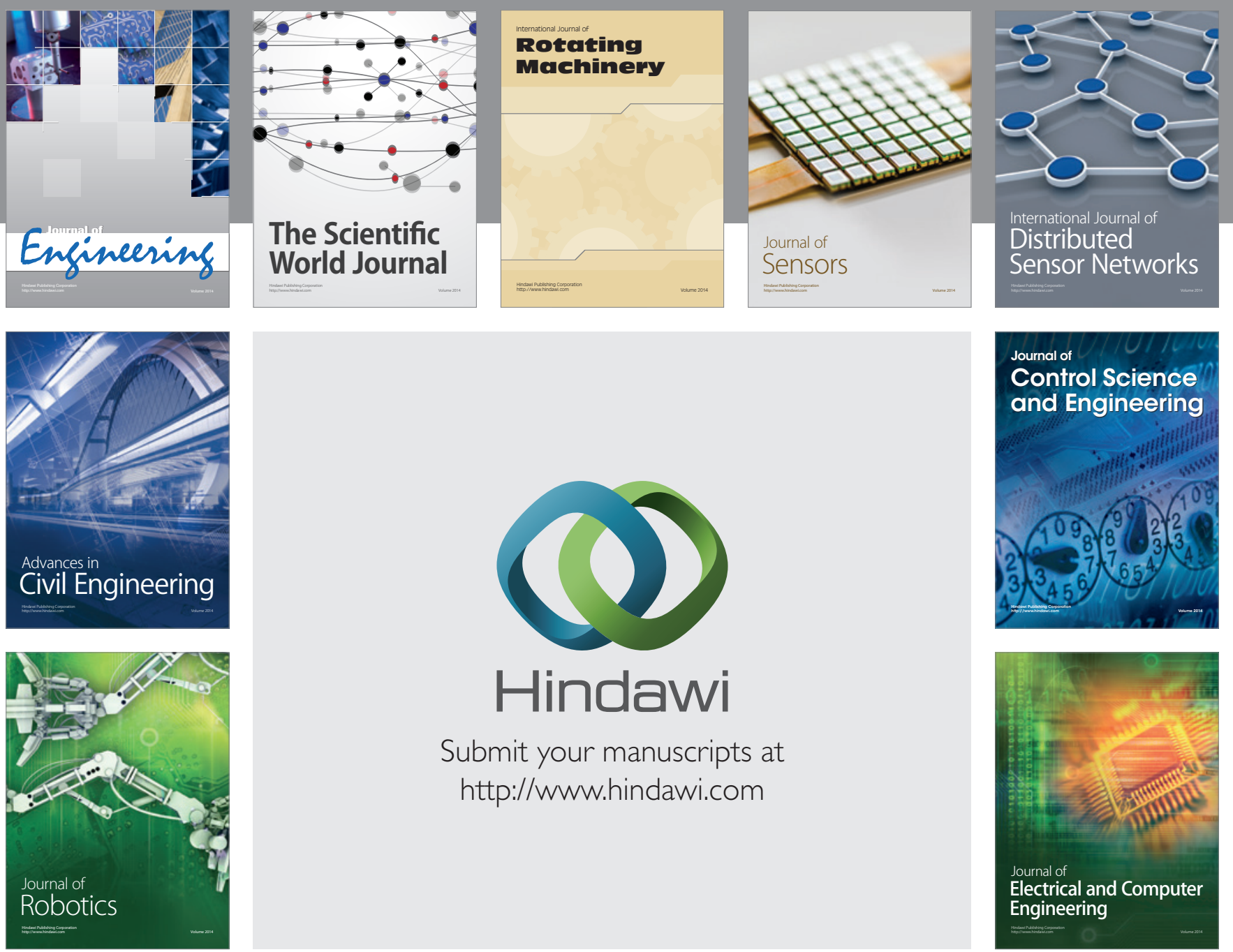

Submit your manuscripts at

http://www.hindawi.com
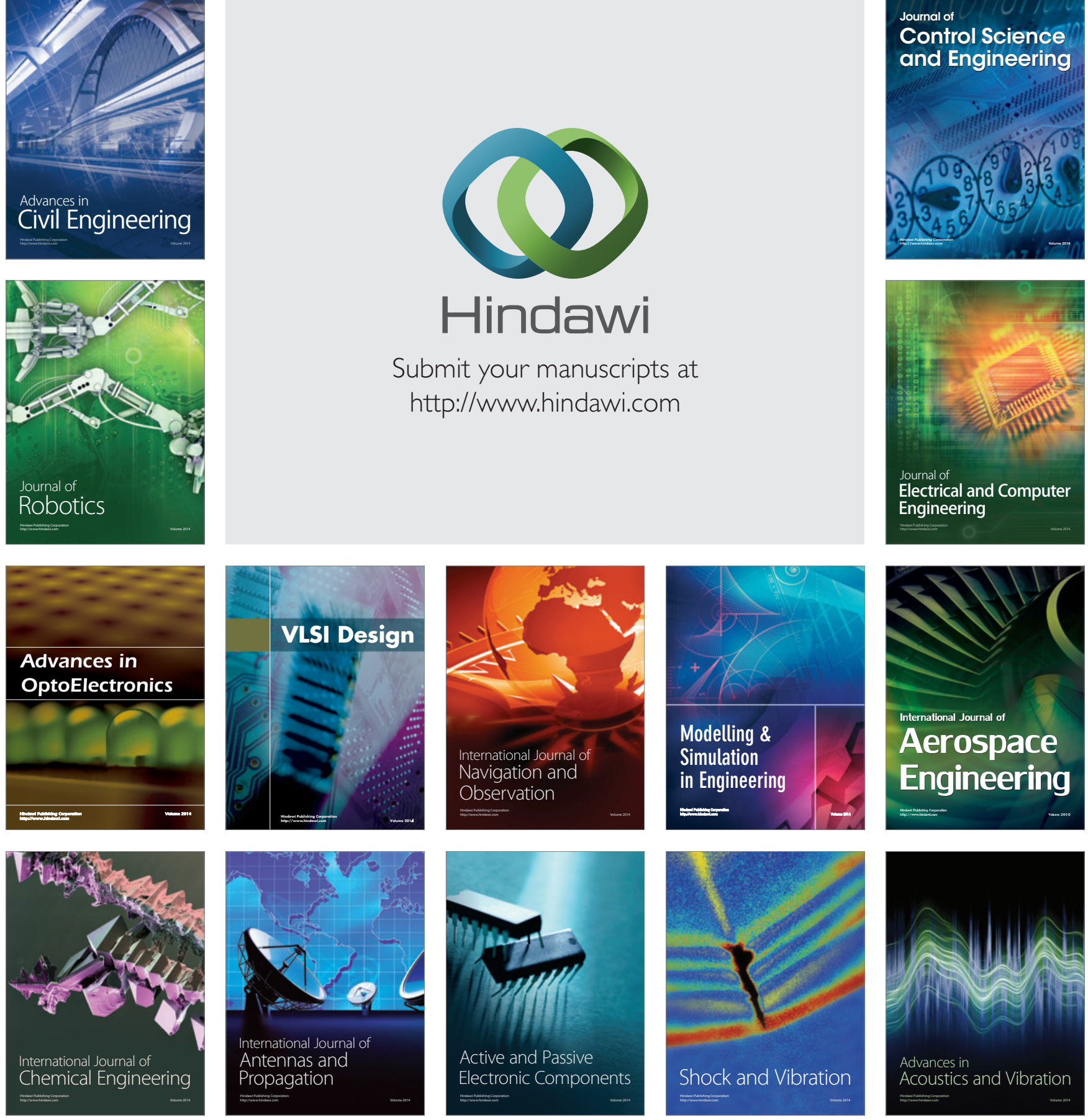